\title{
Registro Andaluz de Arquitectura Contemporánea: metodología y balance del proyecto
}

Plácido González Martínez, Coordinador del RAAC-Centro de Documentación del IAPH

El RAAC supera los límites temporales y de adscripción exclusiva al movimiento moderno
El Registro Andaluz de Arquitectura Contemporánea -RAAC- inició sus trabajos en 2005 con la intención de realizar una aproximación documental extensiva a la arquitectura producida en Andalucía a lo largo del siglo XX. Una labor que daría continuidad a los estudios sobre la arquitectura del Movimiento Moderno en Andalucía -MOMO Andalucía- desarrollados desde el Centro de Documentación del Instituto Andaluz del Patrimonio Histórico, y posteriormente divulgados en colaboración con la Consejería de Obras Públicas y Transportes de la Junta de Andalucía.

En el caso del RAAC, la empresa se mostraba aún más ambiciosa, teniendo en cuenta la variada naturaleza de los objetivos que se marcaba el proyecto:

$\rightarrow$ Reconocer la producción arquitectónica del siglo XX en Andalucía, documentando un numeroso y rico patrimonio que salte los límites temporales establecidos para el MOMO (entre 1925 y 1965), así como la adscripción exclusiva al movimiento moderno, para presentar un panorama cultural enormemente plural, complejo y diverso.

$\rightarrow$ Dar un paso adelante en la defensa de este patrimonio, frecuentemente sometido a trans- formaciones arbitrarias que con demasiada frecuencia conducen a su destrucción. Una labor que requería delimitar previamente los mecanismos a emplear, así como el estudio de la especificidad de las herramientas de protección, para procurar la mayor efectividad al esfuerzo coordinado de catalogación en el que hacer partícipes a las diferentes administraciones.

$\rightarrow$ Transferir los resultados del Registro a la sociedad para promover la justa valoración y protección de esta arquitectura, resaltando su valor patrimonial y reivindicando al mismo tiempo su enorme potencial como elemento estructurante del desarrollo económico y local.

En respuesta a la diversidad de los objetivos a alcanzar, fue necesaria la articulación sinérgica de diferentes organismos e instituciones. El paso inicial fue el establecimiento en 2004 de la colaboración del IAPH con el Grupo de Investigación HUM-666 "Ciudad, patrimonio y arquitectura contemporánea en Andalucía" de la Universidad de Sevilla, para el desarrollo de las labores de documentación. De forma simultánea, el Servicio de Protección de la Dirección General de Bienes Culturales -DGBC- de la Junta de Andalucía sumó sus esfuerzos al proyecto promoviendo los trabajos de protección; 


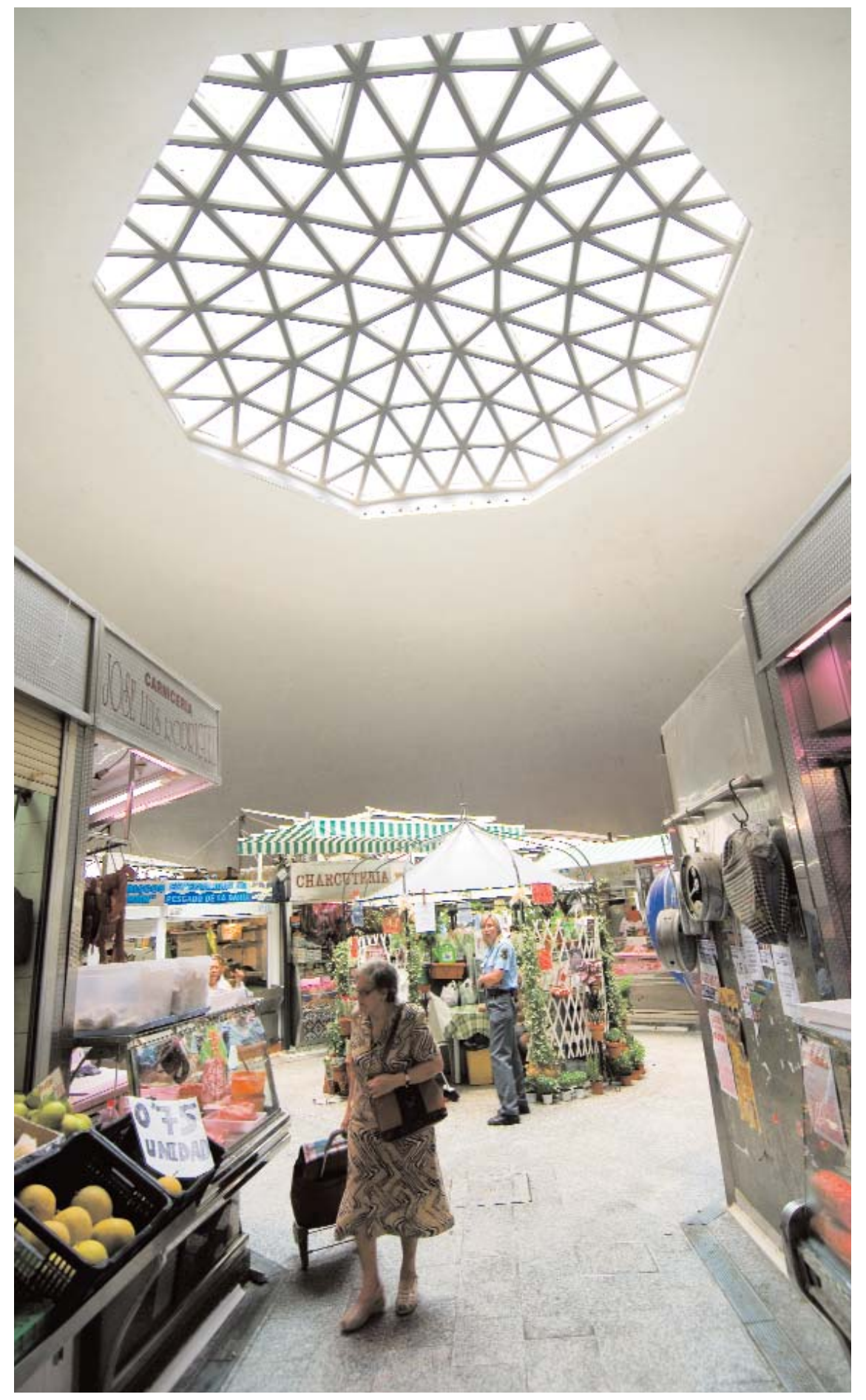

Mercado de Abastos de Algeciras

(Cádiz), diseñado en 1936 por Eduardo

Torroja Miret y Manuel Sánchez Arcas

UAN CARLOS CAZALLA, TAPH 


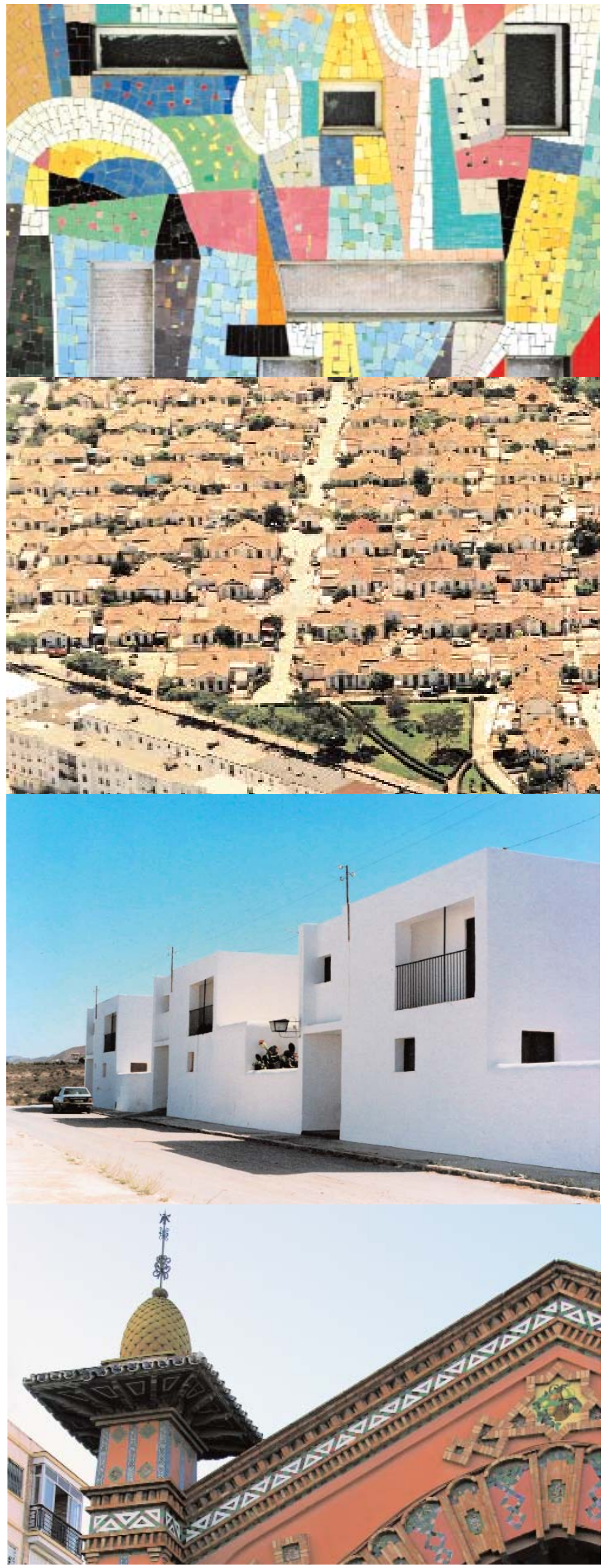

- Viviendas de la ENIRA en Linares (Jaén), obra de Fernando Moreno Barberá del año 1955 /

TIO QUESADA GARCIA

(2) Barrio obrero Reina Victoria en Huelva, diseñado por losé María Pérez Carasa, Gonzalo Aguado y R.H. Morgan entre 1916-1929 / IAPH

(b) Poblado de colonización de Atochares en Nijar (Almería), proyectado por Agustín Delgado Robles en 1959

Miguel Centellas SOler, IAPH

(2) Mercado de Salamanca en Málaga, diseñado en 1925 por Daniel Rubio / ARQYESTUDIO

mientras que las iniciativas de difusión emprendidas desde el Centro de Documentación del IAPH se han potenciado con la cooperación de la Dirección General de Arquitectura y Vivienda de la Junta de Andalucía.

Estructurado el necesario armazón institucional, se establecieron los criterios generales del trabajo, con el fin de abordar eficazmente su complejidad

1. Definiendo los límites temporales del estudio para abarcar de forma exclusiva la arquitectura producida entre 1900 y 2000. Esta delimitación se completó con la subdivisión metodológica del siglo en cinco diferentes etapas comprendidas entre hitos históricos que marcaban cambios de curso en la producción de la arquitectura andaluza:

- 1900-1929; momento en el que el peso del eclecticismo y la pujanza del regionalismo, así como los primeros balbuceos de la modernidad, se manifiestan en una producción del todo singular, culminada con la celebración de la Exposición Iberoamericana de 1929

-> 1930-1939; una etapa marcada por la irrupción del movimiento moderno en Andalucía, coincidente en el tiempo con la instauración de la República, y que se cierra con el final de la Guerra Civil y el inicio de la dictadura.

$\rightarrow$ 1940-1959; periodo en el cual el ingenio y la precariedad conviven con la voluntad afirmativa de la arquitectura del poder, en una reconstrucción marcada por el aislamiento político y la autarquía económica, que finaliza con el Plan de Estabilización.

- 1960-1975; momento de despegue económico y de definitiva apertura de la arquitectura española a las influencias provenientes de la esfera internacional, hasta el inicio de la Transición Democrática.

-> 1976-1992; etapa de intensa producción edilicia marcada por el impulso decisivo del 
Estado de las Autonomías, que culmina con los fastos de la Exposición Universal de 1992.

$\rightarrow$ 1993-2000, cuando la resaca de la Expo decanta la producción de arquitectura en Andalucía, revelando su calidad y su carácter referencial para el resto de España.

2. Haciendo extensivo este estudio no solo a obras de arquitectura, sino también a elementos urbanos, paisajísticos y de obra civil. Por este motivo, se eliminaron las limitaciones numéricas iniciales para construir una visión plural de la arquitectura andaluza en el pasado siglo.

3. Estableciendo el nivel de información de los registros a detallar; una decisión estrechamente ligada a la elección del procedimiento de carga y gestión de la información. El Sistema de Información del Patrimonio Histórico de Andalucía -SIPHA- ofrecía la herramienta idónea para este propósito, requiriéndose de cada edificio la cumplimentación de unos datos básicos, relativos a su identificación y descripción, así como a su localización, delimitación e información bibliográfica, documental y gráfica.

Con esta metodología de trabajo se ponían las bases para desarrollar un proceso de selección que requería de especial rigor, estableciendo filtros que redundasen en su objetividad, de manera particular en las etapas más recientes, que constituyen una parte sustancial de los edificios a inventariar. Para ello se puso en marcha un procedimiento de selección vertical (territorial) y decantación horizontal (temática), desarrollado de la manera siguiente:

En primer lugar, mediante la elaboración de listados iniciales de selección, desarrollados a nivel provincial, que se definieron de manera colegiada a través de sucesivas reuniones de trabajo, buscando el establecimiento de índices homogéneos de calidad, equilibrio territorial y distribución temporal. Esta selección fue completada a lo largo de la primavera de 2005, gracias a la participa- ción de los equipos provinciales de trabajo y del Servicio de Protección de la DGBC, incluyendo un total de 900 bienes, cifra que superaba con creces las estimaciones iniciales del proyecto.

La segunda fase contó con las aportaciones de expertos sectoriales externos que realizaron lecturas transversales de valoración de la selección inicial. Hasta julio de 2006 las aportaciones sobre arquitectura de las explotaciones agrícolas, de la industria, del paisaje, poblados de colonización, arquitectura funeraria, rehabilitación, así como sobre el caso específico de la arquitectura del primer cuarto de siglo, se sumaron a las revisiones temáticas realizadas por los propios equipos de trabajo, contribuyendo a decantar la selección hasta entonces efectuada y ampliándola hasta un total de 1100 bienes.

El proceso de selección se hacía eco al mismo tiempo del objetivo de la protección, que requería esfuerzos redoblados de rigor y concreción. Una tarea en la que la aportación de los expertos externos, el trabajo de documentación de los equipos provinciales y la implicación del Servicio de Protección de la DGBC ha culminado en la selección de un conjunto de 240 edificios sobre los cuales se procede a la redacción de expedientes de catalogación genérica.

En la actualidad, próximos a culminarse los trabajos de documentación y catalogación, el RAAC entra en la fase de difusión, considerada de importancia vital por su incidencia directa en la ciudadanía. Esta última etapa se centra en la elaboración de diferentes productos que hagan accesibles los resultados de este ingente trabajo, tanto al investigador especializado como a las instituciones y el público general.

Los primeros esfuerzos del Centro de Documentación del IAPH se han encaminado a la creación de la Base de Datos de Arquitectura Contemporánea de Andalucía [http://www.juntadeandalu- cia.es/cultura/iaph/bdac/start.do], presentada con ocasión del VI congreso nacional Docomomo Ibérico, celebrado en Cádiz en abril de 2007, y en la que se ofrece un amplio sistema de referencia a nivel general y específico sobre este patrimonio. También para su volcado en la red, los resultados del RAAC han sido transferidos al Inventario de Arquitectura del siglo XX del proyecto ARCHS.XX-SUDOE para España, las regiones del sur de Francia y Gibraltar [http://www. archxx-sudoe.org], que fue presentado en la sede del IAPH el pasado mes de junio de 2007.

Otro de los frentes de actuación incide en el fomento del conocimiento directo de estos bienes, y con ese propósito el Centro de Documentación del IAPH emprende la realización de diferentes itinerarios culturales sobre arquitectura contemporánea en Andalucía, el primero de los cuales aparece en el presente número del Boletín PH trazando un recorrido por la arquitectura del turismo en el tramo de la Carretera del Mediterráneo comprendido entre las ciudades de Cádiz y Málaga. Posicionándose en el aspecto patrimonial de la controvertida cuestión del desarrollo territorial, el RAAC sirve de vehículo para la puesta en valor de una arquitectura de contrastada calidad que subsiste en entornos urbanos y paisajísticos hoy día lamentablemente banalizados.

La multiplicidad de iniciativas plantea un reto complejo, de muchas facetas y escalas, que culminará con la realización de una exposición itinerante y la edición de una publicación al efecto, en cuyo contenido y diseño se trabaja actualmente en colaboración con la Dirección General de Arquitectura y Vivienda de la Junta de Andalucía. Mostrando la enorme diversidad y riqueza de esta topografía de la arquitectura contemporánea andaluza que el Registro se ha encargado de explorar, se desencadenará la imprescindible sensibilización hacia el valor de esta importantísima manifestación cultural: una conciencia patrimonial, en definitiva, que abrigue a esta arquitectura y ofrezca soporte a la que aún está por venir. 


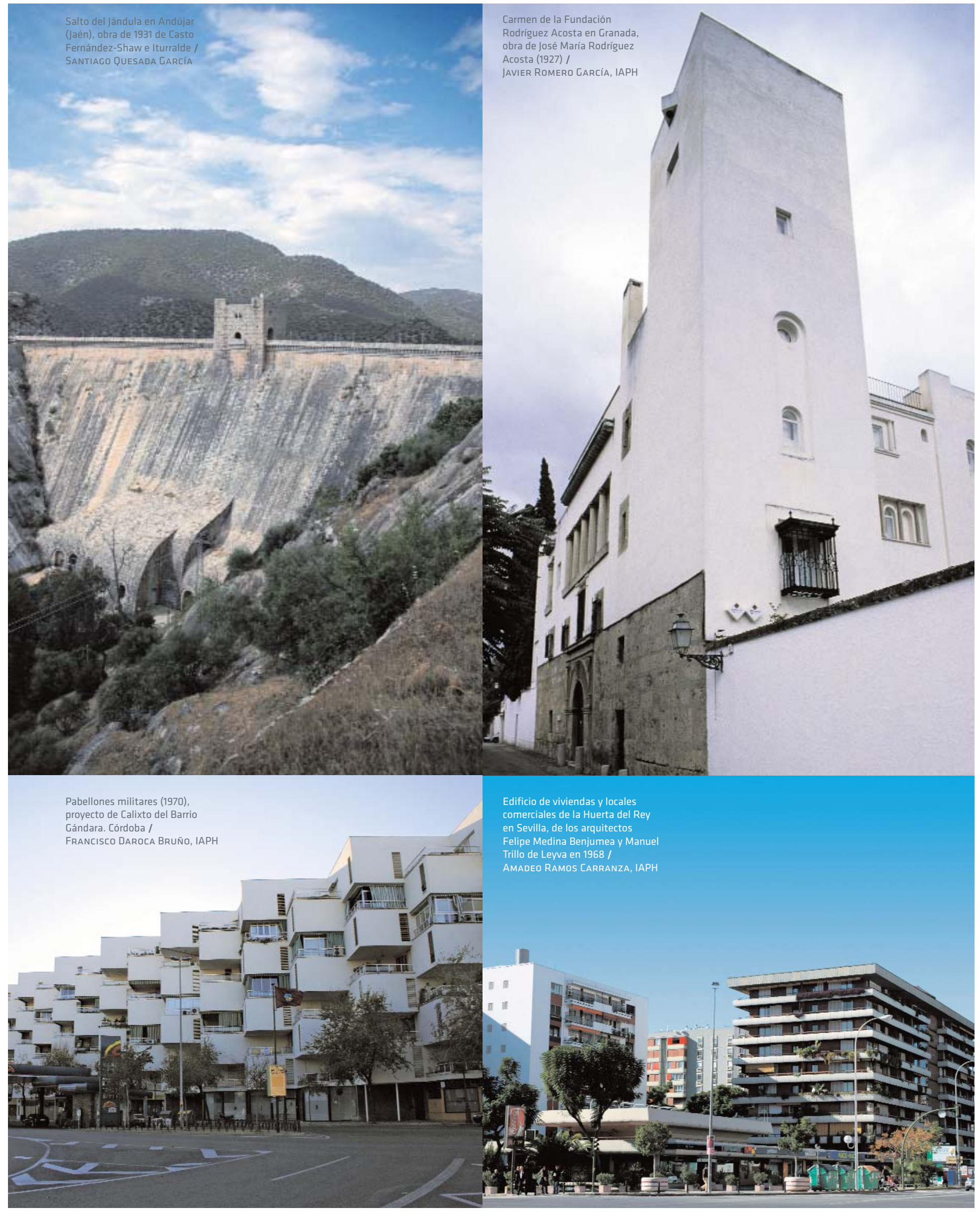




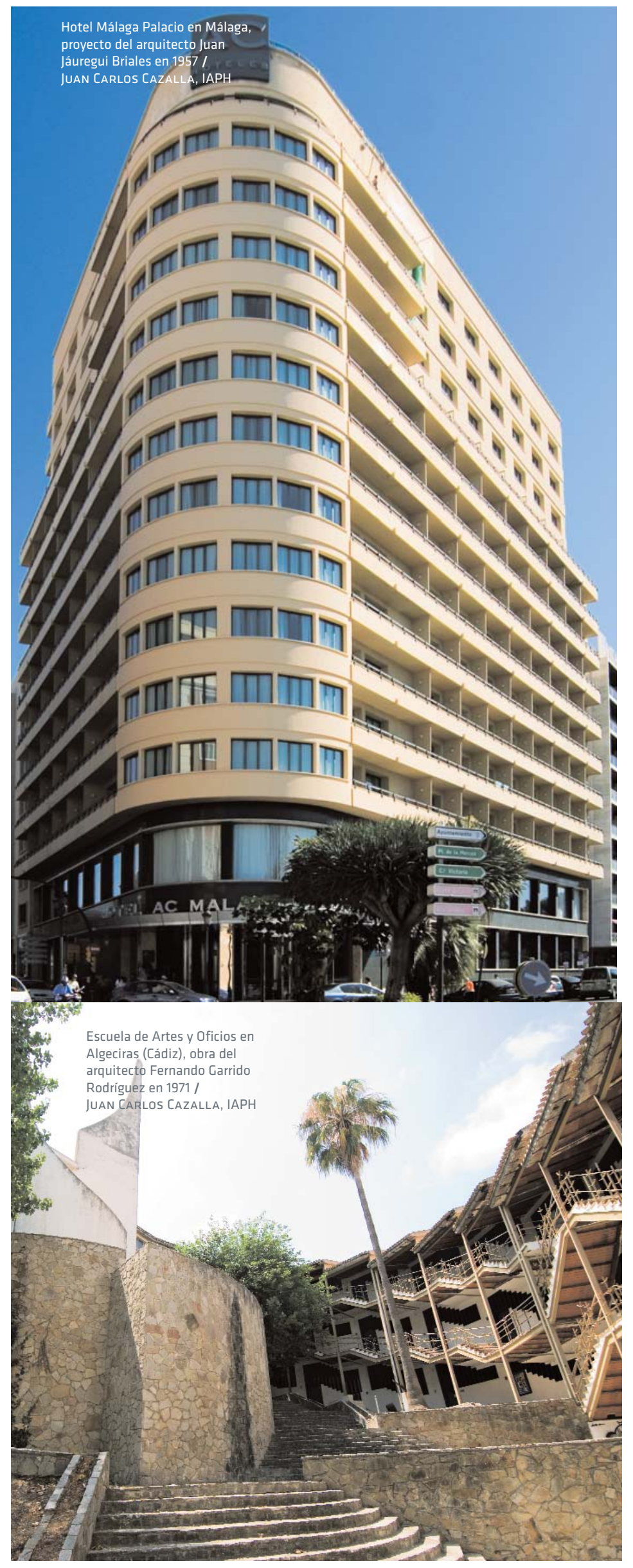

\section{Proyecto RAAC}

COMISIÓN DE SEGUIMIENTO

Román Fernández-Baca Casares. Director del IAPH Víctor Pérez Escolano. Director del Grupo de Investigación HUM666. Universidad de Sevilla Mª Carmen Ladrón de Guevara Sánchez. Jefa del Centro de Documentación del IAPH

Juan Manuel Becerra García. Jefe del Servicio de Protección de la DGBC

Ramón Pico Valimaña. Grupo de Investigación HUM666. Universidad de Sevilla

Valle Muñoz Cruz. Centro de Documentación del IAPH Plácido González Martínez. Centro de Documentación del IAPH

Manuela Gómez Rodríguez. Centro de Documentación del IAPH (hasta 2006)

\section{EQUIPOS DE TRABAJO}

Almería: Miguel Centellas Soler, Alfonso Ruiz García, Pablo García-Pellicer López

Cádiz: Ramón Pico Valimaña, José Domingo Lago

Martín, Cristina López-Lago González, María Llerena Iniesta

Córdoba: Francisco Daroca Bruño, Lorenzo Illanes Ortega, Rafael Zurita Serrano

Granada: Antonio Jiménez Torrecillas, Alberto García Moreno, David Arredondo Garrido

Huelva: Ángel López Macías

Jaén: Santiago Quesada García, Ana Belén Ruiz, Javier Nieto Cano

Málaga: Mar Loren Méndez, Rafael Delacour Jiménez, Sebastián Galafate Domínguez, Ana $\mathrm{M}^{\mathrm{a}}$ Rodríguez Rico, Daniel Pinzón Ayala

Sevilla: Javier Terrados Cepeda, Ignacio Capilla Roncero, Amadeo Ramos Guerra, José Ignacio Sánchez-Cid Endériz, Marta Santofimia Albiñana, Adolfo Gross García

Servicio de Protección de la DGBC. Fuensanta Plata García, Mª Ángeles Pazos Bernal, Florencio Javier Aspas Jiménez

Colaboradores EXPERTOS

Antonio Tejedor Cabrera. Espacios públicos y jardines Manuel Calzada Pérez. Poblados de Colonización Julián Sobrino Simal. Arquitectura de la industria Francisco Javier Rodríguez Barberán. Arquitectura funeraria y cementerios

Fernando Olmedo Granados. Arquitectura de explotaciones agrarias en Andalucía

Ángel Isac Martínez de Carvajal. Arquitectura andaluza del primer tercio del siglo XX

Alberto Villar Movellán. Arquitectura andaluza del primer tercio del siglo XX 


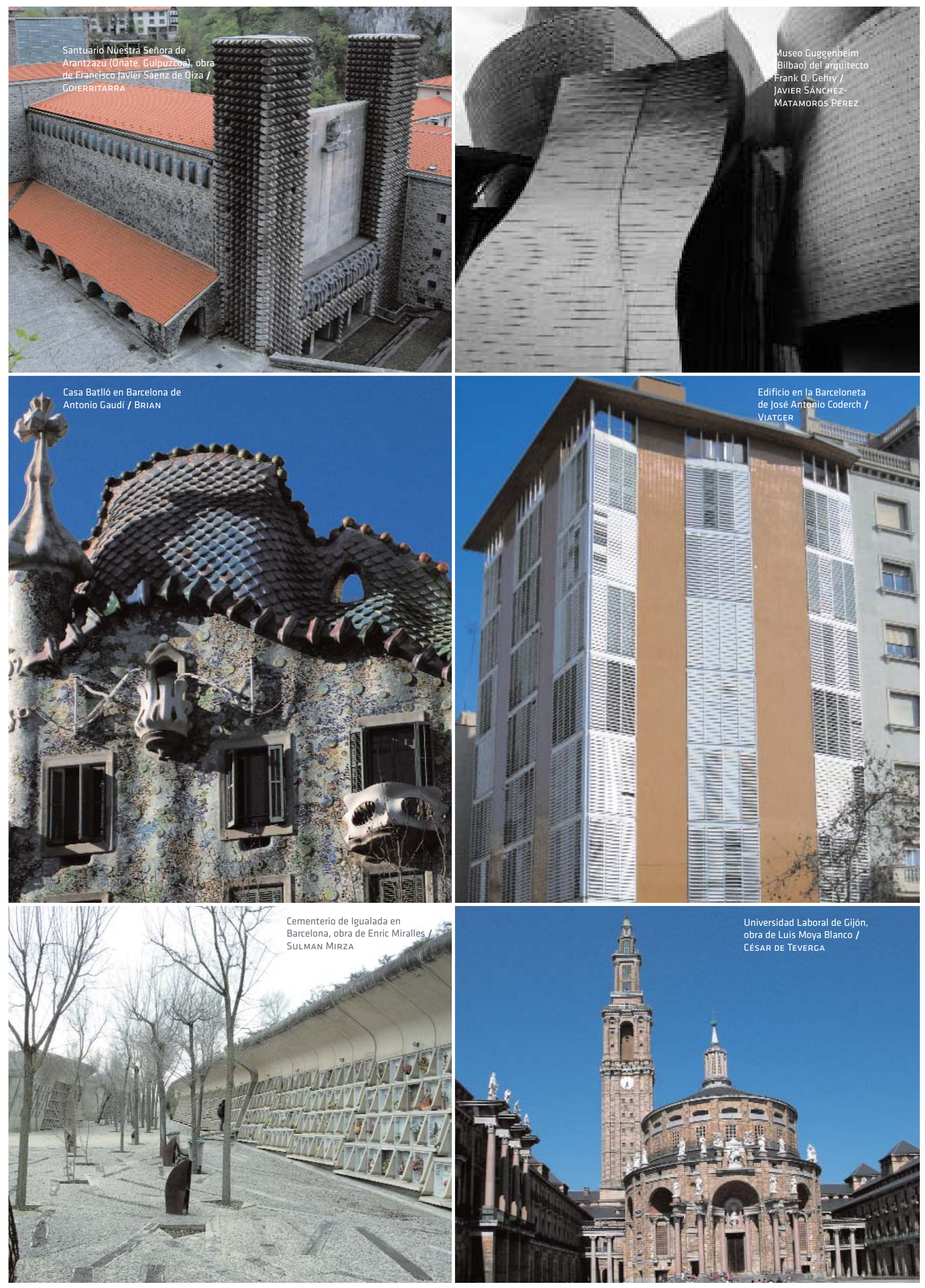

